\title{
A Resting-state Functional Magnetic Resonance Imaging Study of Whole-brain Functional Connectivity of Voxel Levels in Patients With Irritable Bowel Syndrome With Depressive Symptoms
}

\author{
Jie Li, ${ }^{1}$ Ping He, ${ }^{2}$ Xingqi Lu, ${ }^{1}$ Yun Guo, ${ }^{3}$ Min Liu, ${ }^{1}$ Guoxiong Li, ${ }^{3}$ and Jianping Ding ${ }^{1 *}$ \\ ${ }^{I}$ Department of Radiology, The Affiliated Hospital of Hangzhou Normal University, Hangzhou, Zhejiang, China; ${ }^{2}$ Department of Orthodontics, \\ Hangzhou Stomatological Hospital, Hangzhou, Zhejiang, China; and ${ }^{3}$ Department of Gastroenterology, The Affiliated Hospital of Hangzhou \\ Normal University, Hangzhou, Zhejiang; China
}

\section{Background/Aims}

Depressive symptom is one of the most common symptoms in patients with irritable bowel syndrome (IBS), but its pathogenetic mechanisms remain unclear. As a voxel-level graph theory analysis method, degree centrality (DC) can provide a new perspective for exploring the abnormalities of whole-brain functional network of IBS with depressive symptoms (DEP-IBS).

\section{Methods}

$D C$, voxel-wise image and clinical symptoms correlation and seed-based functional connectivity $(F C)$ analyses were performed in 28 DEP-IBS patients, 21 IBS without depressive symptoms (nDEP-IBS) patients and 36 matched healthy controls (HC) to reveal the abnormalities of whole brain FC in DEP-IBS.

\section{Results}

Compared to nDEP-IBS patients and HC, DEP-IBS patients showed significant decrease of DC in the left insula and increase of DC in the left precentral gyrus. The DC's z-scores of the left insula negatively correlated with depression severity in DEP-IBS patients. Compared to nDEP-IBS patients, DEP-IBS patients showed increased left insula-related FC in the left inferior parietal lobule and right inferior occipital gyrus, and decreased left insula-related FC in the left precentral gyrus, right supplementary motor area (SMA), and postcentral gyrus. In DEP-IBS patients, abstracted clusters' mean FC in the right SMA negatively correlated with depressive symptoms.

\section{Conclusions}

DEP-IBS patients have abnormal FC in brain regions associated with the fronto-limbic and sensorimotor networks, especially insula and SMA, which explains the vicious circle between negative emotion and gastrointestinal symptoms in IBS. Identification of such alterations may facilitate earlier and more accurate diagnosis of depression in IBS, and development of effective treatment strategies.

(J Neurogastroenterol Motil 2021;27:248-256)

Key Words

Brain; Depression; Emotions; Irritable bowel syndrome

Received: September 14, 2020 Revised: October 11, 2020 Accepted: December 2, 2020

(.) This is an Open Access article distributed under the terms of the Creative Commons Attribution Non-Commercial License (http://creativecommons. org/licenses/by-nc/4.0) which permits unrestricted non-commercial use, distribution, and reproduction in any medium, provided the original work is properly cited.

${ }^{*}$ Correspondence: Jianping Ding, MD Department of Radiology, The Affiliated Hospital of Hangzhou Normal University, No 126 Wenzhou Street, Gongshu District, Hangzhou, Zhejiang 310015, China Tel: +86-15805819016, E-mail: 598299041@qq.com 


\section{Introduction}

Irritable bowel syndrome (IBS) is a highly recurrent chronic functional gastrointestinal disorder (FGID) with the typical clinical symptoms of recurrent abdominal pain or discomfort, changes in stool characteristics and bowel habits. ${ }^{1}$ The occurrence of IBS is associated with gastrointestinal motility abnormalities, visceral hypersensitivity, intestinal inflammation, and psychological disorders, which can influence IBS symptoms through brain-gut interaction. ${ }^{2}$ Almost half of IBS patients suffer from psychological-psychiatric disorders, such as depression, approximately $20-40 \%$ of patients accompany with depressive symptoms, which seriously affect the quality of life and work efficiency of patients. ${ }^{3,4}$ It has been reported that the combined application of antidepressants can not only effectively improve the mood of IBS patients, but also play a central analgesic effect and weaken visceral sensitivity. ${ }^{5}$ The influence of psychological factors on the intestinal tract may be related to the "brain-gut axis," which is defined as the bidirectional regulatory system, including central nervous system CNS, neuro-endocrineimmune system, hypothalamic-pituitary-adrenal axis, autonomy nervous system, and enteric nervous system. It plays an important role in regulating gastrointestinal motility, visceral sensitivity, braingut peptide secretion, stress response, and cognitive function. ${ }^{6} \mathrm{Ab}-$ normal emotional activities and mental states can change autonomic nervous function through the emotional stress circuit, which mainly include cerebral cortex, limbic system, locus ceruleus, vagus/hypothalamic dorsal nucleus, autonomic nervous system, and enteric nervous system, leading to gastrointestinal dysfunction and visceral perception disorders. ${ }^{7}$ Up to now, the neural mechanism of depressive symptoms in IBS is still poorly understood and remains inconclusive. Hence, to further explore the brain function changes, such as neuronal activity in the local brain region and the strength of functional connectivity (FC) between brain regions in IBS patients with specific psychological symptoms (such as depression) will help us understand the pathophysiological mechanism of the brain-gut interaction of FGID. In clinical terms, it can provide a scientific basis for formulating personalized treatment strategies.

A large number of studies have reported that FGID patients have functional abnormalities in the limbic system and sensorimotor networks, which are partly associated with depressive symptoms. ${ }^{8-13}$ Resting-state functional magnetic resonance imaging (fMRI) can detect the activities of brain neurons in the resting state, which was one of the effective methods to study the neural network mechanism related to brain-gut interaction. Using the amplitude of low- frequency fluctuations ${ }^{14,15}$ and regional homogeneity ${ }^{16}$ analysis methods, the changes in the activity of local brain regions in the prefrontal lobe-limbic system, basal ganglia and default mode network were showed in patients with IBS, which partly associated with depressive symptoms. Studies based on seed-point and wholebrain functional connection analysis methods have shown that IBS patients had disconnection of emotions, limbic system, sensorimotor, and default mode networks, which are partly associated with depressive symptoms. ${ }^{1718}$ Our previous study found that patients with IBS with depressive symptoms (DEP-IBS) have abnormalities in the microstructure of brain regions related to pain and emotion in the prefrontal-limbic circuit, which further causes and aggravates depressive symptoms (or gastrointestinal symptoms) through the intermediation of gastrointestinal symptoms (or depressive symptoms). ${ }^{19}$ It is speculated from the results of the above studies that some regions in the frontal lobe-limbic system is involved not only in the perception of visceral pain, but also in the emotional experience triggered by various stresses in the emotional activity system. It can mediate unpleasantness or depression caused by abdominal pain, and it can also affect the perception of abdominal pain in patients with IBS when depression and other emotional disorders occur. Depression can directly cause the activation of the limbic system, thereby affecting the transmission of visceral pain, leading to visceral hyperesthesia, abdominal pain, and related emotional abnormalities. The results help to understand more clearly why the gastrointestinal symptoms of IBS patients with psychological abnormalities are more serious than those of patients without psychological abnormalities. ${ }^{3}$ The inclusion of psychosocial factors may be crucial to elucidate the neural network mechanism of IBS.

The above studies focus on local spontaneous brain activity or analyze neural networks between selected brain regions based on a prior assumption. In recent years, graph-based analysis methods applied to the analysis of complex neural networks, such as the attribute analysis of "small-world" brain networks. ${ }^{20}$ According to graph theory, the network considered as a pair relationship between system elements, which consists of a set of edges connecting a set of nodes. ${ }^{21}$ Network analysis provides a new conceptual framework for the study of network biology in neurodegenerative and psychosocial diseases. ${ }^{21,22}$ Among measures based on the voxel level, degree centrality (DC) refers to the number of connections that are strongly correlated with nodes of a given voxel or binary graph. It can analyze the brain at the voxel level and quantify the importance of a node to other parts of the brain, thus avoiding the bias caused by the selection of brain regions based on prior assumptions. ${ }^{23}$ The DC method can reflect the topological properties of nodes or brain 
regions in the whole brain network and is a measure to describe the importance of network nodes. ${ }^{20,23}$ In the present study, the DC method for whole-brain FC analysis of voxel-level based on a graph theoretical approach and a secondary seed-based $\mathrm{FC}$ analysis were used to comprehensively explore the intrinsic abnormality of the whole-brain functional network in DEP-IBS patient from the node and network attributes. We hypothesized that long-term negative emotions in IBS patients lead to abnormal FC in certain brain areas, especially those involved in emotional, limbic, basal ganglia and sensorimotor networks, resulting in abnormal regulation of visceral pain perception (such as visceral hypersensitivity), further aggravate gastrointestinal symptoms and negative emotions, thus forming a vicious circle.

\section{Materials and Methods}

\section{Subjects}

Forty-nine right-handed patients with IBS were recruited from the digestive clinic, as well as $36 \mathrm{HC}$ with matched age, sex and educational level, were enrolled from the physical examination center. This study protocol conforms to the ethical guidelines of the 1975 Declaration of Helsinki as reflected in a priori approval by the institution's human research committee (Approval No. HZNU20160427), and written informed consent was obtained from all participants before data collection.

All patients fulfilled the following criteria: (1) diagnoses were confirmed on the basis of the Rome III symptom criteria, ${ }^{24}$ (2) were not taken antidepressant and prokinetic drugs before enrollment, (3) were without any previous or current neurological disorders and head injury, and (4) were right-handed. A complete medical history taking and physical examination, blood profiles, and colonoscopy were performed in all patients. HCs were no history of chronic pain, bowel disturbance, or visceral disease. Other criteria were the same as those applied to the patients. The enrolled IBS patients were further divided based on the presence or absence of depressive symptoms according to the 17-item Hamilton Depression Rating Scale (HAMD-17), ${ }^{25}$ forming 2 groups: DEP-IBS (mean \pm SD, $12.75 \pm 1.71$ ) and IBS with non-depressive symptoms (nDEPIBS) (mean $\pm \mathrm{SD}, 3.71 \pm 1.38$ ). HAMD-17 is the most commonly used scale for clinical assessment of depressive status. The total score is 24 points. Patients with a score higher than 7 were considered depressed. Higher scores indicate more severe depression. Their demographic and clinical information are shown in Table 1.

\section{Magnetic Resonance Imaging Data Acquisition}

Brain imaging was performed by a whole-body $3 \mathrm{~T}$ GE Discovery MR-750 scanner and an 8-channel head coil (GE Healthcare, Waukesha, WI, USA). All subjects were instructed to remain still and awake, close their eyes, and breathe steadily during the image acquisition process. Special non-magnetic headphones and spongy pads were used to reduce noise and limit head movement. Functional images were acquired using blood oxygenation level dependent-gradient echo-echo planar imaging sequence with parameters: flip angle, $90^{\circ}$; repetition time, $2000 \mathrm{msec}$; echo time, 30 msec; matrix, $64 \times 64$; field of view, $192 \mathrm{~mm} \times 192 \mathrm{~mm}$; inplane resolution, $3.44 \mathrm{~mm} \times 3.44 \mathrm{~mm}$; 37 slices covering the whole brain; and slice thickness, $4 \mathrm{~mm}$. The resting-state sequence scanning time was 8 minutes, and 240 volumes were transversely acquired. After the resting-state scan, structural images were also collected for each subject, which acquired using the spoiled gradient-recalled pulse sequence with parameters: flip angle, $8^{\circ}$; TR, 8.16 msec; echo time, 3.18 msec; matrix, $256 \times 256$; thickness, $1 \mathrm{~mm}$; and 176 sagittal slices covering the whole brain. In addition, rou-

Table 1. Demographic and Clinical Characteristics of Participants

\begin{tabular}{|c|c|c|c|c|}
\hline Variables & DEP-IBS $(\mathrm{n}=28)$ & nDEP-IBS $(\mathrm{n}=21)$ & $\mathrm{HC}(\mathrm{n}=36)$ & $P$-values \\
\hline Female/Male & $11 / 17$ & $9 / 12$ & $10 / 26$ & $0.456^{\mathrm{a}}$ \\
\hline Age (yr) & $36.36 \pm 7.31$ & $32.29 \pm 9.96$ & $31.67 \pm 8.85$ & $0.087^{\mathrm{b}}$ \\
\hline Education (years) & $11.82 \pm 3.09$ & $12.95 \pm 2.58$ & $13.28 \pm 3.32$ & $0.164^{\mathrm{b}}$ \\
\hline Duration of IBS (months) & $20.64 \pm 9.01$ & $18.57 \pm 4.00$ & - & $0.331^{\mathrm{c}}$ \\
\hline GSRS & $42.11 \pm 11.76$ & $23.43 \pm 5.42$ & - & $<0.001^{\mathrm{c}}$ \\
\hline HAMD-17 & $12.75 \pm 1.71^{\mathrm{d}}$ & $3.71 \pm 1.38^{\mathrm{e}, \mathrm{f}}$ & $0.42 \pm 0.77$ & $<0.001^{\mathrm{b}}$ \\
\hline
\end{tabular}

IBS, irritable bowel syndrome; DEP-IBS, IBS patients with depressive symptoms; nDEP-IBS, IBS patients without depressive symptoms; HC, healthy controls; GSRS, gastrointestinal symptoms rating scale; HAMD, Hamilton depressive rating scale.

Data are shown as mean $\pm \mathrm{SD}$.

${ }^{a} \chi^{2}$; ${ }^{b} P$-values of one-way ANOVA and post hoc comparisons (DEP-IBS vs $\mathrm{HC},{ }^{\mathrm{d}} \mathrm{P}<0.001 ; \mathrm{nDEP}$ vs $\mathrm{HC},{ }^{\mathrm{e}} \mathrm{P}<0.001 ; \mathrm{DEP}$ vs $\mathrm{nDEP},{ }^{\mathrm{f}} \mathrm{P}<0.001$ ); ${ }^{\mathrm{c}} \mathrm{Unpaired} t$ test. 
tine clinical MRI sequences such as T1-weighted image and T2weighted-fluid-attanuated inversion recovery (T2-FLAIR) were collected to exclude anatomical abnormalities and organic brain disease.

\section{Magnetic Resonance Imaging Data Processing}

Prior to data processing, T1- and T2-FLAIR weighted images of each participant were examined by 2 senior radiologists, and 2 subjects with abnormal signs such as mass lesions and cerebral infarction were excluded.

Data preprocessing was implemented using the toolbox of Data Processing and Analysis for (Resting-State) Brain Imaging (DPABI) (http://www.restfmri.net/forum) ${ }^{26}$ in Statistical Parametric Mapping software version 8 (available from URL: http://www. fil.ion.ucl.ac.uk/spm/software/spm8/). The software runs in MATLAB R2013b (The Mathworks, Natick, MA, USA). The Digital Imaging and Communications in Medicine (DICOM) format of MRI data of all subjects was first converted to a standard format (Neuroimaging Informatics Technology Initiative). The image data of the first 10 time points of the resting state scan were eliminated to enable the subjects to adapt to the scanning environment. Slicetiming was performed to eliminate the interval scanning time difference. The head movement signal was corrected and the head movement information of each subject was obtained. Subjects whose head movements were shifted by more than $3 \mathrm{~mm}$ in the $x, y$, and $z$ directions or rotated by more than $3^{\circ}$ were excluded (all the subjects met this standard). Gray matter, white matter, and cerebrospinal fluid of each individual structural image were segmented, and then resampled $(3 \mathrm{~mm} \times 3 \mathrm{~mm} \times 3 \mathrm{~mm})$ in the Montreal Neurological Institute (MNI) space. Detrending was applied to remove the linear drift of the baseline signal. The data were bandpass filtered (0.01-0.08 Hz) to eliminate physiological noise. The head motion parameters, cerebrospinal fluid signal, white matter signal, and mean value signal of the whole brain were regressed as covariables to improve the accuracy of the results.

Based on preprocessing, the $\mathrm{DC}$ was calculated using DPABI software in a voxel-wise manner with a threshold $r>0.25 .^{23}$ Based on the graph theory method, each voxel in the whole brain was defined as a node, and the linear correlation of neural activity signals between voxels was regarded as an edge. For each subject, the correlation between any voxel pairs was calculated within the DPABI brain default template. Then, the whole-brain functional network was constructed by thresholding each correlation, and the DC value at the individual level was divided by the mean value of the whole brain DC, to obtain the standardized DC value. To improve the normality, Fisher's $R$ to $Z$ and $Z$ transformation were used to convert the individual related data into Fisher's $Z$ value. Finally, the $Z$-valued DC distribution map of each subject was obtained. The weighted DC graph was obtained by summation of the positive correlation coefficients ( $Z$ values) between a given voxel and all other voxels. The resulting images were smoothed with $6 \mathrm{~mm}$ full-width half maximum.

\section{Statistical Methods}

We analyzed demographic and clinical data using a one-way analysis of variance (ANOVA), $\chi^{2}$-test, and post hoc unpaired $t$ tests. DC comparisons among the 3 groups were performed using a one-way analysis of covariance (ANCOVA) in DPABI with nuisance covariates, including age, sex, education level, and head movement parameters. These areas were extracted as a mask. A 2-sample post hoc $t$ test within this mask was performed between each pair of the 3 groups (AlphaSim correction, $P<0.05$ ). Partial correlation analysis (age, sex, education level, and head movement as covariables) was used to assess the correlation between DC values of brain regions with intergroup differences and HAMD and gastrointestinal symptoms rating scale (GSRS) scores. Finally, brain regions associated with HAMD scores were used as seed points (spherical regions with a radius of $6 \mathrm{~mm}$ centered on the peak coordinates of MNI in the DC map) for FC analysis based on seed points. Besides, to explore the relationship between seed-based FC abnormalities and depressive symptoms in DEP-IBS patients, the partial correlation analyses between FC abnormalities clusters and HAMD scores of the DEP-IBS patients were also performed. As we know, IBS has typical sex differences, ${ }^{27}$ so we further use a 2 (sex) $\times 2$ (group) mixed model ANOVA statistical method to analyzed the data results according to sex.

\section{Results}

\section{Demographic Characteristics and Clinical Results}

Demographic details of all subjects and clinical characteristics of patients are summarized in Table 1 . There were no statistically significant differences among the 3 groups in sex, age, and education level. Both patient groups had higher HAMD scores $(P<$ 0.001) than did the HC group. Moreover, the GSRS and HAMD scores were significantly higher in the DEP-IBS group than in the nDEP-IBS group $(P<0.001)$. The 2 patient groups did not differ significantly in IBS disease duration $(P=0.330)$. No significant sex specificity was found in the gastrointestinal symptoms and 
Table 2. Brain Regions Showing Significant Differences in Degree Centrality Among 3 Groups

\begin{tabular}{|c|c|c|c|c|c|c|}
\hline \multirow{2}{*}{ Brain area } & \multirow{2}{*}{$\mathrm{R} / \mathrm{L}$} & \multicolumn{3}{|c|}{ MNI coordinates } & \multirow{2}{*}{$\begin{array}{l}\text { Cluster size } \\
\text { (voxels) }\end{array}$} & \multirow{2}{*}{$\begin{array}{c}\text { Peak } \\
F \text {-values }\end{array}$} \\
\hline & & $\mathrm{x}$ & $\mathrm{y}$ & $\mathrm{z}$ & & \\
\hline Insula & $\mathrm{L}$ & -33 & 18 & 0 & 33 & 11.37 \\
\hline Medial superior frontal gyrus & $\mathrm{L}$ & -6 & 51 & 12 & 36 & 9.23 \\
\hline Precentral gyrus & $\mathrm{L}$ & -54 & 12 & -18 & 53 & 12.71 \\
\hline
\end{tabular}

R, right; L, left; MIN, Montreal Neurological Institute.

One-way ANCOVA results $(P<0.05$, AlphaSim multiple comparisons corrected) showed that the brain regions with differences in degree centrality values among the irritable bowel syndrome with depressive symptoms, irritable bowel syndrome without depressive symptoms, and healthy controls.

A
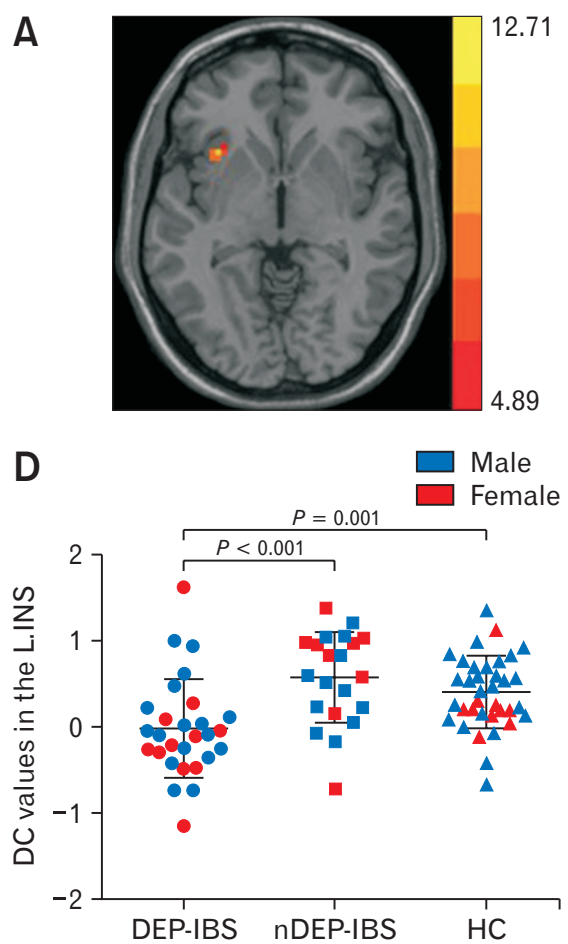

B

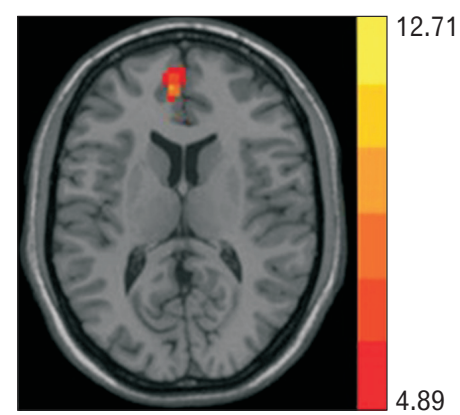

C

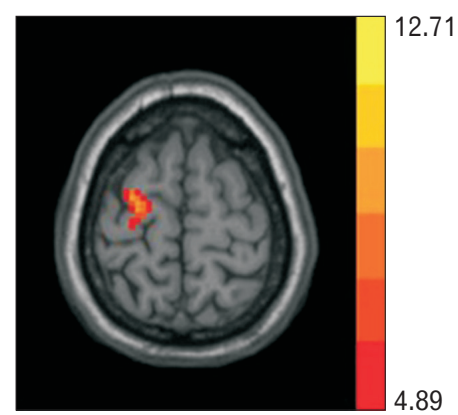

E

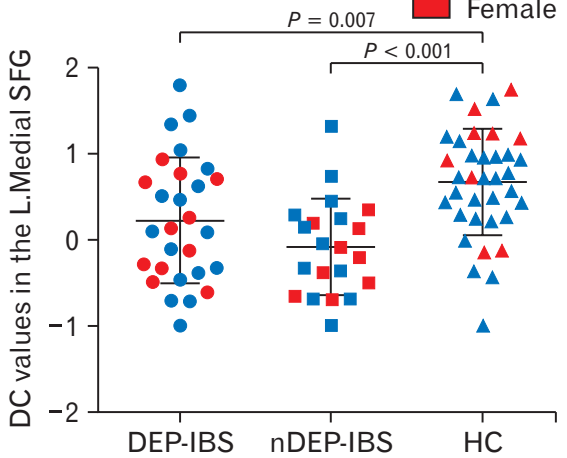

F

4.89
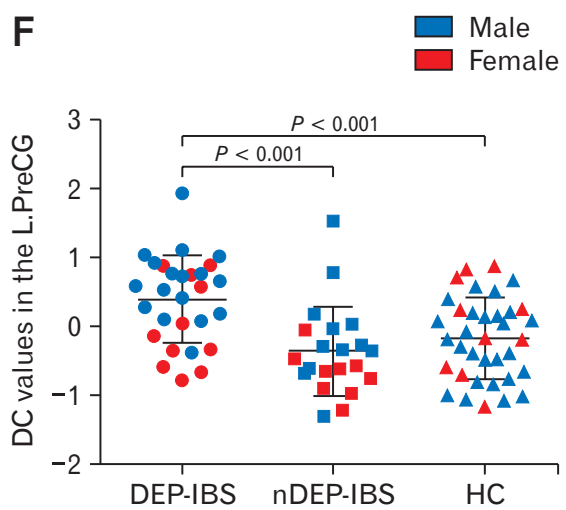

Figure 1. One-way analysis of covariance (ANCOVA) and post hoc 2-sample $t$ test results of degree centrality among irritable bowel syndrome (IBS) with depressive symptoms (DEP-IBS) group, IBS without depressive symptoms (nDEP-IBS) group, and healthy controls $(\mathrm{HC})$ group $(P<0.05$, Alphasim correction). Brain regions with different degree centrality (DC) values among groups and sex distribution in each group: (A) left insula, (B) left medial superior frontal gyrus, and (C) left precentral gyrus. The distribution and comparison of DC values of brain regions (A-C) in the 3 groups (D-F). L.INS, left insula; L.medial SFG, left medial superior frontal gyrus; L.PreCG, left precentral gyrus.

depressive symptoms of the 2 patient groups (DEP-IBS group: GSRS $P=0.673$, HAMD $P=0.956$; nDEP-IBS group: GSRS $P=0.521, \mathrm{HAMD} P=0.895)$.

\section{Degree Centrality Analysis}

One-way ANCOVA results $(P<0.05$, AlphaSim multiple comparisons corrected) showed that the brain regions with differences in DC values among the 3 groups included the left insula (Table 2 and Fig. 1A), the left medial superior frontal gyrus (Table 2 and Fig. 1B), and the left precentral gyrus (Table 2 and Fig. 1C).
Post hoc 2-sample $t$ test comparison results (Fig. 1D-F) showed that compared with nDEP-IBS patients and HC, DEP-IBS patients showed reduced DC in the left insula (Fig. 1D), and increased the DC in the left precentral gyrus (Fig. 1F). There was no significant difference in the $\mathrm{DC}$ value of the above 2 brain regions between $\mathrm{nDEP}-\mathrm{IBS}$ group and $\mathrm{HC}$ group. The $\mathrm{DC}$ value of left medial superior frontal gyrus in both groups was significantly lower than that in the HC group, while there was no statistically significant difference between the 2 groups of IBS patients (Fig. 1E). The ANOVA results showed that the DC values in the left insula and 


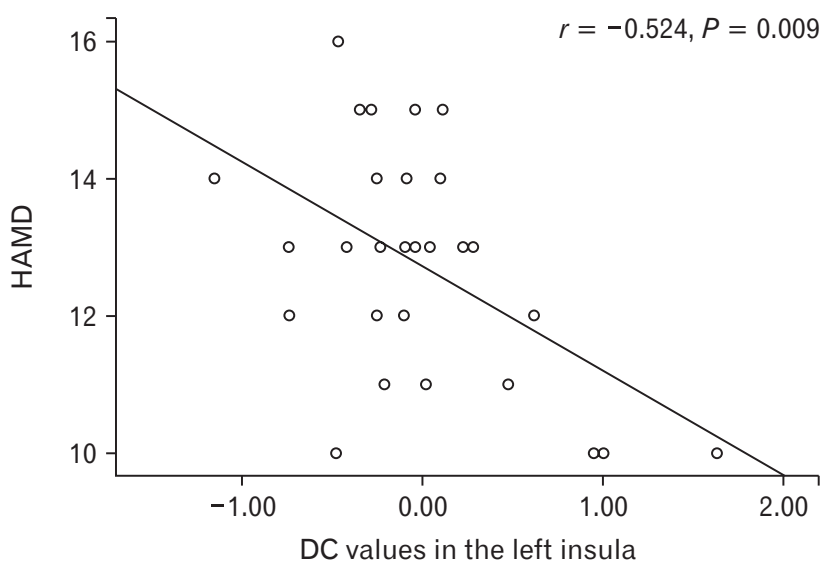

Figure 2. Scatter plots depicting partial correlation between degree centrality (DC) and the clinical depression severity for irritable bowel syndrome with depressive symptoms patients. HAMD, Hamilton depression rating scale.

A
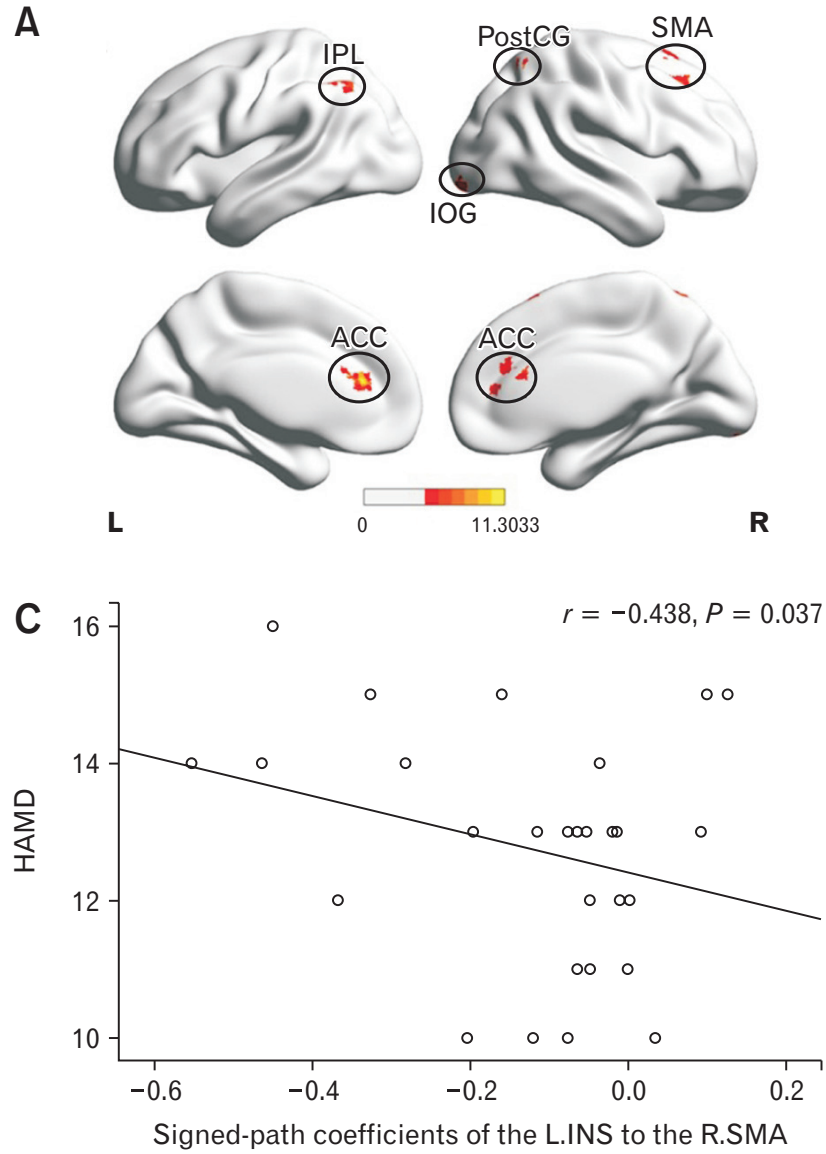

in the left medial superior frontal gyrus did not have a significant group-sex interaction effect $(F=0.924, P=0.401 ; F=1.203$, $P=0.306)$. There was a significant group-sex interaction effect on the DC values in the left precentral gyrus ( $F=4.800, P=$ $0.011)$. In the 2 patient groups, the $\mathrm{DC}$ values in the left precentral gyrus of female patients was significantly lower than that of male patients in each group (DEP-IBS group: $P=0.011$; nDEP-IBS group: $P=0.040$ ). There was no sex difference in the $\mathrm{DC}$ values of the left precentral gyrus in the $\mathrm{HC}$ group $(P=0.243)$.

\section{Correlation Between Degree Centrality Value and Hamilton Depression Rating Scale Scores}

Partial correlation analysis showed that the DC value of the left insula of DEP-IBS patients was significantly negatively correlated with HAMD score $(r=-0.524, P=0.009)$, as shown in Figure 2 .

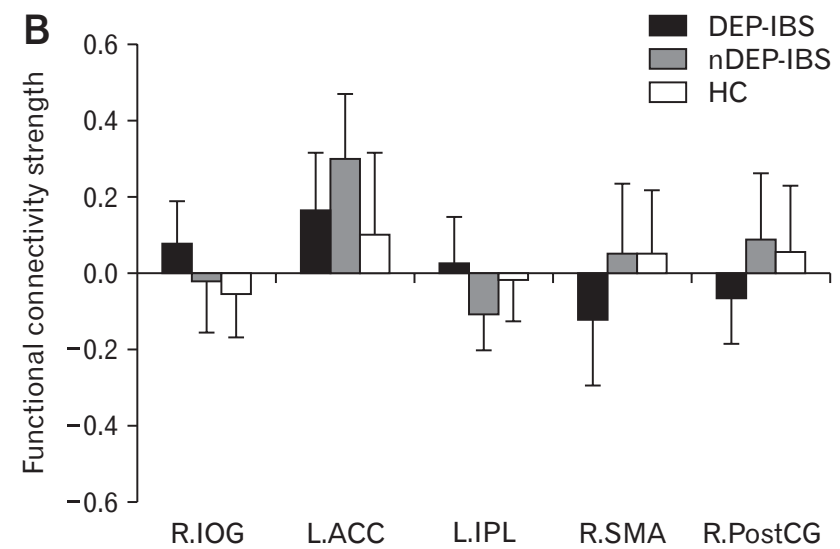

Figure 3. One-way analysis of covariance (ANCOVA) (A) and post hoc 2-sample $t$ test (B) results of left insula seed functional connectivity analyses differences among irritable bowel syndrome (IBS) with depressive symptoms (DEP-IBS) group, IBS without depressive symptoms (nDEP-IBS) group, and healthy controls (HC) group. AlphaSim multiple comparisons corrected is $P<0.05$. Scatter plots depicting partial correlation between the left insula-related functional connectivity in the right supplementary motor area (SMA) and the Hamilton depression rating scale (HAMD) score for DEP-IBS patients (C). L, left; R, right; IPL, inferior parietal lobule; PostCG, postcentral gyrus; IOG, inferior occipital gyrus; ACC, anterior cingulate cortex; INS, insula. 
Since no sex difference was found in the left insula DC values in the DEP-IBS group, the results of this correlation analysis were not further analyzed by sex.

\section{Secondary Seed-based Functional Connectivity Analysis}

The left insula cluster (peak MNI: $x=-33, y=18, z=0$ ) was further used as a seed for the secondary seed-based FC analysis. Figure 3A shows the one-way ANCOVA results, and Figure 3B shows the post hoc 2-sample $t$ test results. Compared with HC, patients with DEP-IBS showed increased left insula-related FC in the right inferior occipital gyrus, and decreased left insula-related FC in the right supplementary motor area (SMA) and the right postcentral gyrus. The above differences were not shown between the $\mathrm{nDEP}-\mathrm{IBS}$ and $\mathrm{HC}$ groups. Compared with the $\mathrm{nDEP}-\mathrm{IBS}$ group, patients with DEP-IBS showed the same pattern of FC changes as described above, and there were additional increased left insula-related FC in the left inferior parietal lobule and decreased left insula-related FC in the bilateral anterior cingulate cortex (ACC) (Fig. 3B). In addition, FC intensity between left insula and right SMA negatively correlated with the score of HAMD in DEP-IBS patients $(r=-0.438, P=0.037$ ) (Fig. 3C).

\section{Discussion}

To the best of our knowledge, this study is the first to explore voxel-level whole-brain FC abnormalities in DEP-IBS patients using both DC and seed-based FC approaches. The main findings were as follows: (1) compared with nDEP-IBS patients and HC, DEP-IBS patients showed decreased DC in the left insula and increased DC in the left precentral gyrus. The DC values of the left medial superior frontal gyrus in both IBS groups were significantly lower than that in the HC group; (2) the DC abnormalities in the left insula correlated with depression severity in the DEP-IBS patients; and (3) changes of the left insula-related FC in the right SMA, the right postcentral gyrus, the right inferior occipital gyrus, the inferior parietal lobule and the bilateral ACC were further observed. In particular, the left insula-related FC abnormalities in the right SMA of DEP-IBS patients were associated with HAMD scores.

The insula, as part of the limbic system, is involved in the integration of visceral sensory functions and is responsible for sending pain signals to the frontal cortex, playing a crucial role in modulation of visceral sensation and movement, and pain-related emotional processing. ${ }^{28} \mathrm{~A}$ large number of functional MRI (fMRI) studies have reported the functional and structural abnormalities of the insula in IBS patients, and there were significant correlations with variables such as gastrointestinal symptoms, anxiety and depression symptoms. ${ }^{29-31}$ A positron emission tomography-computed tomography study based on fluorine-18-deoxyglucose found that after controlling gastrointestinal symptoms, the metabolic abnormalities of the insular and prefrontal cortex (PFC) of patients with functional dyspepsia remained, and there was a significant correlation with anxiety/depressive symptoms. ${ }^{32}$ These results suggested that anxiety/depression may interfere with glucose metabolism in the insula and prefrontal lobes of FGID patients. A recent study found abnormal insular activity in patients with IBS in response to visceral stimulation and endocrine stress, which is associated with alexithymia. ${ }^{33}$ Our study showed that DEP-IBS patients had decreased DC in the left insula and negatively correlated with the HAMD score. This negative correlation suggest that decreased DC was associated with the severity of pain-related negative emotions. A metaanalysis of task-state fMRI studies related to pain stimuli found that brain regions positively related to pain involve ACC, $\mathrm{PFC}$, primary and secondary somatosensory cortex, insular cortex, and thalamus. The recognition of pain intensity activates the ventral pathway from the insular cortex to the bilateral PFC, while the recognition of pain space involves the dorsal pathway from the posterior parietal cortex to the right dorsolateral PFC. Both of the above tasks lead to the activation of ACC. ${ }^{34}$ The ACC is closely connected with the SMA, premotor and primary motor cortex, which together form a module that generates and transmits the final decision about the nature of the sensory features of the discriminated pain stimuli. A secondary seed-based FC analysis in our study further revealed that changes in the left insula-related FC exist in patients with chronic visceral pain, including ACC, somatosensory cortex (postcentral gyrus) and posterior parietal cortex. In particular, abnormalities in the FC between insula and SMA were associated with depression severity in DEPIBS patients. Growing evidence also suggests that SMA is not only a motor brain region, but also plays an important role in the integration of affective and cognitive functions. ${ }^{35}$ Patients with depression show disrupted functional integration in SMA, which may be due to psychomotor retardation, and is considered one of the vital neurophysiological indicators of depression. ${ }^{36}$ Numerous studies have found that functional changes in SMA networks can be used as predictors of antidepressant treatment outcomes, and may serve as a biomarker of treatment response. ${ }^{37-39}$ Our previous study found that DEP-IBS patients had decreased gray matter volume in the insula and sensorimotor cortex. ${ }^{19}$ Therefore, we speculate that the insula and SMA may be the key pathogenesis of DEP-IBS patients, and 
may provide a certain guiding value for the formulation of clinical treatment strategies.

The precentral gyrus is a sensorimotor region, consisting of the premotor cortex and the SMA, receiving information projected from the basal ganglia. Bhatt et $\mathrm{al}^{40}$ found that the $\mathrm{FC}$ between the precentral gyrus and caudate nucleus was enhanced in IBS patients. The basal ganglia is involved in the integration of information with the cortical, thalamic regions and 3 specific pain processing regions, which is an important part of the "pain matrix." 41 The caudate nucleus is the largest nucleus in the basal ganglia, which can produce analgesic effects when stimulated. ${ }^{42}$ Our study showed that increased DC in the left precentral gyrus in DEP-IBS patients, which may be related to the depression that further strengthens the patients' perception of visceral pain. More interestingly, our data showed that female patients with IBS had lower DC than male patients in the left precentral gyrus but not in the HC group, suggesting precentral's ability in integrating and receiving visceral hypersensitivity information can be influenced by sex.

This study showed decreased DC in the left medial superior frontal gyrus in both IBS groups. MRI studies in chronic pain patients more consistently reported decreased gray matter density in the medial PFC ( $\mathrm{mPFC}$ ) and decreased functional connections between $\mathrm{mPFC}$ and other brain regions. ${ }^{43,44}$ The activity of PFC is related to anti-hyperalgesia. ${ }^{45}$ Studies suggest that $\mathrm{mPFC}$ can release endogenous opioids during tension pain. ${ }^{46}$ Therefore, the decreased DC in $\mathrm{MPFC}$ may be related to the weakened function of the pain suppression system.

This study has several shortcomings. First, the sample size of IBS is relatively small, which may affect the credibility and universality of the results. Second, the study did not analyze other concomitant symptoms or factors, such as anxiety symptoms or traumatic experiences. Thirdly, the HAMD score of DEP-IBS patients was within the range of 10 to 17 points, which only belonged to the state of mild depression and did not meet the clinical diagnostic criteria for depression. In the future, the sample size should be further expanded to refine the concomitant symptoms and sex differences of IBS patients, and comparative analysis should be conducted with patients with simple depression for mutual verification.

The pathogenesis of depressive symptoms in IBS was associated with dysfunctions of fronto-limbic circuit and sensorimotor network, which were reflected by the abnormal FC pattern of these networks in our study. Especially, the insula and SMA, could be taken as a characteristic feature for depression in IBS. Identification of such alterations may facilitate earlier and more accurate diagnosis of depression in IBS, and development of effective treatment strategies.
Acknowledgements: Our team thanks all patients and healthy volunteers for their participation.

Financial support: This work was supported by the Social Development Project of Zhejiang Public Welfare Technology Research (Grant No. 2015C33292 and LGF20H180016), and the Project of Zhejiang Medical and health Science and Technology (Grant No. 2020KY710).

\section{Conflicts of interest: None.}

Author contributions: Jie Li, Ping He, and Xingqi Lu contributed to data analysis and in completing the manuscript; Guoxiong $\mathrm{Li}$ and Jianping Ding reanalyzed data and improved the language of the manuscript; Yun Guo and Guoxiong Li provided clinical information; and Jie Li and Min Liu collected the MRI data. All of the above authors contributed to data acquisition and conducted the experiments.

\section{References}

1. Mayer EA. Clinical practice. Irritable bowel syndrome. N Engl J Med 2008;358:1692-1699.

2. Quigley EMM. The gut-brain axis and the microbiome: clues to pathophysiology and opportunities for novel management strategies in irritable bowel syndrome (IBS). J Clin Med 2018;7:6.

3. Talley NJ, Howell S, Poulton R. The irritable bowel syndrome and psychiatric disorders in the community: is there a link? Am J Gastroenterol 2001;96:1072-1079.

4. Muscatello MR, Bruno A, Scimeca G, Pandolfo G, Zoccali RA. Role of negative affects in pathophysiology and clinical expression of irritable bowel syndrome. World J Gastroenterol 2014;20:7570-7586.

5. Ruepert L, Quartero AO, de Wit NJ, van der Heijden GJ, Rubin G, Muris JW. Bulking agents, antispasmodics and antidepressants for the treatment of irritable bowel syndrome. Cochrane Database Syst Rev 2011:CD003460.

6. Price DD, Zhou Q, Moshiree B, Robinson ME, Verne GN. Peripheral and central contributions to hyperalgesia in irritable bowel syndrome. J Pain 2006;7:529-535.

7. Zhang QE, Wang F, Qin G, et al. Depressive symptoms in patients with irritable bowel syndrome: a meta-analysis of comparative studies. Int J Biol Sci 2018;14:1504-1512.

8. Lee C, Doo E, Choi JM, et al. The increased level of depression and anxiety in irritable bowel syndrome patients compared with healthy controls: systematic review and meta-analysis. J Neurogastroenterol Motil 2017;23:349-362.

9. Mudyanadzo TA, Hauzaree C, Yerokhina O, Architha NN, Ashqar HM. Irritable bowel syndrome and depression: a shared pathogenesis. Cureus 2018;10:e3178.

10. Levy RL, Olden KW, Naliboff BD, et al. Psychosocial aspects of the 
functional gastrointestinal disorders. Gastroenterology 2006;130:14471458.

11. Zhou G, Qin W, Zeng F, et al. White-matter microstructural changes in functional dyspepsia: a diffusion tensor imaging study. Am J Gastroenterol 2013;108:260-269.

12. Drossman DA, Ringel Y, Vogt BA, et al. Alterations of brain activity associated with resolution of emotional distress and pain in a case of severe irritable bowel syndrome. Gastroenterology 2003;124:754-761.

13. Qi R, Liu C, Weng Y, et al. Disturbed interhemispheric functional connectivity rather than structural connectivity in irritable bowel syndrome. Front Mol Neurosci 2016;9:141.

14. Qi R, Liu C, Ke J, et al. Intrinsic brain abnormalities in irritable bowel syndrome and effect of anxiety and depression. Brain Imaging Behav 2016;10:1127-1134.

15. Ma X, Li S, Tian J, et al. Altered brain spontaneous activity and connectivity network in irritable bowel syndrome patients: a resting-state fMRI study. Clin Neurophysiol 2015;126:1190-1197.

16. Ke J, Qi R, Liu C, et al. Abnormal regional homogeneity in patients with irritable bowel syndrome: a resting-state functional MRI study. Neurogastroenterol Motil 2015;27:1796-1803.

17. Qi R, Ke J, Schoepf UJ, et al. Topological reorganization of the default mode network in irritable bowel syndrome. Mol Neurobiol 2015;53:6585-6593.

18. Weng Y, Qi R, Liu C, et al. Disrupted functional connectivity density in irritable bowel syndrome patients. Brain Imaging Behav 2017;11:18121822.

19. Li J, Yuan B, Li G, et al. Convergent syndromic atrophy of pain and emotional systems in patients with irritable bowel syndrome and depressive symptoms. Neurosci Lett 2020;723:134865.

20. Xia M, He Y. Functional connectomics from a "big data" perspective. Neuroimage 2017;160:152-167.

21. Petersen SE, Sporns O. Brain networks and cognitive architectures. Neuron 2015;88:207-219.

22. de Haan W, van Straaten ECW, Gouw AA, Stam CJ. Altering neuronal excitability to preserve network connectivity in a computational model of Alzheimer's disease. PLoS Comput Biol 2017;13:e1005707.

23. Zuo XN, Ehmke R, Mennes M, et al. Network centrality in the human functional connectome. Cereb Cortex 2012;22:1862-1875.

24. Drossman DA, Dumitrascu DL. Rome III: new standard for functional gastrointestinal disorders. J Gastrointestin Liver Dis 2006;15:237-241.

25. Hamilton M. A rating scale for depression. J Neurol Neurosurg Psychiatry 1960;23:56-62.

26. Yan CG, Wang XD, Zuo XN, Zang YF. DPABI: data processing \& analysis for (resting-state) brain imaging. Neuroinformatics 2016;14:339-351.

27. Anbardan SJ, Daryani NE, Fereshtehnejad SM, Taba Taba Vakili S, Keramati MR, Ajdarkosh H. Gender role in irritable bowel syndrome: a comparison of irritable bowel syndrome module (Rome III) between male and female patients. J Neurogastroenterol Motil 2012;18:70-77.

28. Nagai M, Kishi K, Kato S. Insular cortex and neuropsychiatric disorders: a review of recent literature. Eur Psychiatry 2007;22:387-394.

29. Jiang Z, Dinov ID, Labus J, et al. Sex-related differences of cortical thickness in patients with chronic abdominal pain. PLoS One 2013;8:e73932.
30. Piché M, Chen JI, Roy M, Poitras P, Bouin M, Rainville P. Thicker posterior insula is associated with disease duration in women with irritable bowel syndrome (IBS) whereas thicker orbitofrontal cortex predicts reduced pain inhibition in both IBS patients and controls. J Pain 2013;14:1217-1226.

31. Blankstein U, Chen J, Diamant NE, Davis KD. Altered brain structure in irritable bowel syndrome: potential contributions of pre-existing and disease-driven factors. Gastroenterology 2010;138:1783-1789.

32. Nan J, Liu J, Mu J, et al. Brain-based correlations between psychological factors and functional dyspepsia. J Neurogastroenterol Motil 2015;21:103-110.

33. Kano M, Muratsubaki T, Yagihashi M, et al. Insula activity to visceral stimulation and endocrine stress responses as associated with alexithymia in patients with irritable bowel syndrome. Psychosom Med 2020;82:2938.

34. Oshiro Y, Quevedo AS, McHaffie JG, Kraft RA, Coghill RC. Brain mechanisms supporting discrimination of sensory features of pain: a new model. J Neurosci 2009;29:14924-14931.

35. Nachev P, Kennard C, Husain M. Functional role of the supplementary and pre-supplementary motor areas. Nat Rev Neurosci 2008;9:856-869.

36. Yan CG, Chen X, Li L, et al. Reduced default mode network functional connectivity in patients with recurrent major depressive disorder. Proc Natl Acad Sci USA 2019;116:9078-9083.

37. Korgaonkar MS, Goldstein-Piekarski AN, Fornito A, Williams LM. Intrinsic connectomes are a predictive biomarker of remission in major depressive disorder. Mol Psychiatry 2020;25:1537-1549.

38. Sahib AK, Loureiro JR, Vasavada MM, et al. Modulation of inhibitory control networks relate to clinical response following ketamine therapy in major depression. Transl Psychiatry 2020;10:260.

39. Li CT, Su TP, Wang SJ, Tu PC, Hsieh JC. Prefrontal glucose metabolism in medication-resistant major depression. Br J Psychiatry 2015;206:316-323.

40. Bhatt RR, Gupta A, Labus JS, et al. Altered brain structure and functional connectivity and its relation to pain perception in girls with irritable bowel syndrome. Psychosom Med 2019;81:146-154.

41. Legrain V, Iannetti GD, Plaghki L, Mouraux A. The pain matrix reloaded: a salience detection system for the body. Prog Neurobiol 2011;93:111-124.

42. Price DD. Psychological and neural mechanisms of the affective dimension of pain. Science 2000;288:1769-1772.

43. Yuan C, Shi H, Pan P, et al. Gray matter abnormalities associated with chronic back pain: a meta-analysis of voxel-based morphometric studies. Clin J Pain 2017;33:983-990.

44. Tan Y, Wu X, Chen J, Kong L, Qian Z. Structural and functional connectivity between the amygdala and orbital frontal cortex in burning mouth syndrome: an fMRI study. Front Psychol 2019;10:1700.

45. Seifert F, Bschorer K, De Col R, et al. Medial prefrontal cortex activity is predictive for hyperalgesia and pharmacological antihyperalgesia. J Neurosci 2009;29:6167-6175.

46. Zubieta JK, Smith YR, Bueller JA, et al. Regional mu opioid receptor regulation of sensory and affective dimensions of pain. Science 2001;293:311-315. 